\title{
Trayectorias de equipos directivos en instituciones de educación terciaria tecnológica en países de MERCOSUR
}

\author{
Trajectories of management teams in technological tertiary \\ education institutions in MERCOSUR countries
}

\section{Irma Briasco ${ }^{1}$ \\ Nancy Montes ${ }^{2}$}

\section{Resumen}

En este artículo se presentan los avances del estudio sectorial "La oferta de educación terciaria tecnológica: caracterización del modelo de gestión desde la perspectiva de los estudios comparados" (Briasco et al., 2020) elaborado por investigadores del ámbito universitario de cuatro países de MERCOSUR: Argentina, Brasil, Chile, Paraguay y Uruguay. El foco del artículo despliega el eje referido a trayectorias de los equipos directivos. El conjunto de aspectos señalados como necesarios para la formación aporta a la posibilidad de organizar una agenda formativa que tenga como destinatarios a quienes se desempeñan en estos espacios de trabajo en una oferta que, como se señaló al inicio del trabajo, flexibiliza sus

\footnotetext{
${ }^{1}$ Doctora en Educación por la Universidad Nacional de Córdoba. Desde 1995 a 2018 Especialista en Educación Técnico Profesional para Iberoamérica de la Organización de Estados Iberoamericanos. Desde 2007 docente e investigadora en la Universidad Pedagógica Nacional, Argentina. Email: irma.briasco@unipe.edu.ar

2 Docente e investigadora del Programa Educación, Conocimiento y Sociedad del Área Educación de la Facultad Latinoamericana de Ciencias Sociales. Especialista de la Organización de Estados Iberoamericanos. Email: nmontes@oei.org.ar
} 
propuestas para responder a los desafíos sociales y formativos del mundo contemporáneo.

Palabras clave: MERCOSUR; terciario tecnológico; trayectorias formativas.

\begin{abstract}
This paper shows an advance of the sector study "The offer of technological tertiary education: characterization of the management model from the perspective of comparative studies" (Briasco et al., 2020) prepared by university researchers from four MERCOSUR countries: Argentina, Brazil, Chile, Paraguay and Uruguay. The focus of the article unfolds the axis referring to the trajectories of the management teams. The set of aspects indicated as necessary for training contributes to the possibility of organizing a training agenda aimed at those who work in these work spaces in an offer that, as indicated at the beginning of the work, makes their proposals more flexible to respond to the social and formative challenges of the contemporary world.
\end{abstract}

Keywords: MERCOSUR; technological tertiary; formative trajectories.

\title{
1. Introducción
}

Este artículo presenta una de las líneas desplegadas en el trabajo de investigación realizado en el marco de la convocatoria NEIES (2018). El objeto del estudio es la caracterización de la oferta de los institutos tecnológicos (CINE 5) de cinco de los países que integran el MERCOSUR. El estudio se desarrolló a partir de tres ejes de trabajo, una descripción de los sistemas de educación técnica superior existentes en cada país con uso de fuentes secundarias; un abordaje de micro nivel para caracterizar los modelos institucionales y un tercer eje que hizo foco en las trayectorias formativas de los equipos directivos. Este texto desarrolla el último de los ejes, si bien incluye algunas referencias necesarias para describir esta oferta a fines de enmarcar el análisis.

El texto da cuenta de las decisiones metodológicas y de los marcos conceptuales para realizar el trabajo de campo y el análisis de las trayectorias 
formativas y profesionales de los integrantes de los equipos directivos. Por último, se identifican los hallazgos de la investigación, dejando formulados temas de discusión referidos a la formación de estos equipos de conducción. Se espera así realizar un aporte en un espacio de vacancia en la construcción de políticas de formación para este segmento de profesionales, no siempre atendido por políticas de formación específicas.

\section{Antecedentes}

Este artículo se basa en un estudio, cuyo propósito fue, en primer lugar, caracterizar la situación de la oferta y, en segundo término, construir una mirada desde la perspectiva institucional en el marco de las políticas de Educación y Formación Técnica y Profesional (EFTP). Siguiendo a UNESCO (2015), la EFTP se define como:

[...] la expresión «enseñanza y formación técnica y profesional» (en adelante EFTP) comprende la enseñanza, la formación y la adquisición de destrezas relativas a una gran variedad de sectores ocupacionales, actividades de producción, servicios y medios de subsistencia. La EFTP, como parte del aprendizaje a lo largo de toda la vida, puede impartirse en los niveles secundario, postsecundario y superior, e incluye el aprendizaje en el trabajo y la formación permanente y el desarrollo profesional que pueden conducir a la obtención de certificaciones. La EFTP incluye asimismo una gran variedad de posibilidades de adquisición de destrezas en función de los contextos nacionales y locales. Aprender a aprender, el desarrollo de aptitudes en materia de lectoescritura y de cálculo y las aptitudes transversales y para la ciudadanía forman parte integral de la EFTP. (p. 5)

El informe de OREALC/UNESCO (2013) afirma que esta definición tuvo una implementación disímil a nivel de los países y recupera distintas indagaciones que muestran que los sistemas educativos establecieron alcances diferenciados para este tipo de formación. Para algunos países de América Latina y el Caribe, la EFTP es una orientación de la educación secundaria, mientras que para otros es una modalidad de educación superior impartida específicamente en institutos técnicos. 
El punto de partida es, entonces, el reconocimiento de la heterogeneidad y complejidad que la EFTP que se expresa en modelos organizacionales y pedagógicos diferenciados, vigentes en los cinco países que forman parte de este estudio. Dicha heterogeneidad se expresa tanto en relación con la estructura, como con la institucionalidad y los modos de gobierno del sistema.

Asimismo, el mencionado estudio de UNESCO señala que los sistemas nacionales de educación superior han experimentado una fuerte expansión durante las últimas décadas producto del aumento continuo de la participación de la población en este nivel educativo. En respuesta a una demanda cada vez más masiva y diversa, los sistemas amplían y diversifican también su oferta mediante la conformación de sistemas crecientemente diferenciados en su base institucional. Por lo tanto, la heterogeneidad propia de la EFTP suma complejidad al entramarse con el dinamismo y diversificación que asume el nivel superior. Esta oferta, a diferencia de la que se imparte en universidades se caracteriza por una mayor vinculación con ámbitos laborales y profesionales más dinámicos que responden o intentan responder a demandas emergentes de los mercados de trabajo de los países de la región.

Por esa razón, se consideró relevante incorporar en el estudio en clave comparada, una dimensión pocas veces abordada relativa a las trayectorias formativas de los equipos directivos, dado que una parte importante de los modelos institucionales también se explica (o se sostiene) por el rol de sus equipos directivos $y$, en esa responsabilidad, los perfiles de quienes los integran resultan de interés para las acciones formativas que las instituciones desarrollan. Como se verá en el apartado 4, muchos de los perfiles que tienen a su cargo las tareas de dirección y gestión en estas instituciones provienen del ámbito empresarial o de inserciones técnico profesionales que aportan saberes y capacidades necesarias para el tipo de propuestas que tienen a su cargo.

Para el estudio de los temas relativos a las trayectorias formativas se partió del relato de los directivos entrevistados, el análisis se complementa indagando las particularidades del vínculo que tiende a establecerse entre las biografías profesionales (Alliaud, 2004; Rivas Flores, 2007), los recorridos formativos y las 
experiencias en el ejercicio del cargo de conducción. Desde esta perspectiva se considera la conformación de los equipos directivos de las instituciones estudiadas, las trayectorias de sus integrantes y la dinámica de trabajo, a fin de componer un análisis que aporte elementos para el diseño de políticas de formación y para la elaboración de propuestas de actualización permanente.

De acuerdo con la bibliografía especializada que ha analizado la formación docente $y$, en particular la correspondiente a los cargos y equipos directivos y al propio relevamiento realizado en el marco de este estudio, no existen ofertas específicas de formación de grado que aborden la formación en gestión directiva de instituciones educativas, en el mejor de los casos se trata de orientaciones, más recientemente algunas licenciaturas en gestión educativa incluyen estos tópicos, pero no siempre teniendo como horizonte de inserción a la educación superior sino más bien a la educación obligatoria. En cambio, las propuestas para el desarrollo profesional de estos perfiles adopta la forma de cursos de posgrado de menor o mayor duración que abordan temas de gestión de las instituciones o de formación de equipos directivos (OEI, 2017; Terigi, 2010; Veleda, 2016, Weinstein, 2014). Otra constatación es que existe poca investigación o disponibilidad de estudios que aborden este tópico para el nivel superior. De hecho, la bibliografía existente se enfoca mayormente sobre la gestión de instituciones que ofrecen los niveles de enseñanza considerados obligatorios en los países de la región.

Cabe plantear algunos aspectos implícitos en la noción de trayectoria y que resultan de utilidad al momento de pensar en el perfil de los equipos de conducción. En principio, hablar de trayectorias implica poner el foco en las personas y en sus recorridos, tanto en lo que hace a la formación inicial y continua como también a la inserción profesional, los cuales no son necesariamente lineales ni continuos. Interesa señalar que la inserción laboral constituye también, para los especialistas en formación docente un ámbito de formación, a veces con mayor peso que la formación inicial (Alliaud, 2010). Se aprende a ser docente o directivo de una institución educativa siendo docente o directivo, en diálogo con prácticas institucionales que tienen a su vez rasgos específicos dependiendo de diversos factores: historia de las instituciones, sector de gestión, culturas institucionales, 
tipos de organización, programas, entre otros. Por otra parte, trabajar sobre las trayectorias implica una dimensión temporal en la vida de las personas que refiere a esos recorridos a lo largo del tiempo, hacia atrás y hacia adelante. En el campo educativo se ha instalado la demanda de "formación continua, permanente" y de modo más amplio, "para toda la vida". Además de esta dimensión, según Bourdieu, las trayectorias se inscriben en una "superficie social" que explica, condiciona, habilita o restringe los caminos y las opciones posibles (Bourdieu, 1997).

Los estudios sobre trayectorias se han constituido en una opción metodológica en aquellas investigaciones que se proponen recuperar los recorridos seguidos por los individuos en cuanto a lo profesional y laboral. Analizar, comprender y reflexionar sobre trayectorias supone definir los recorridos 0 itinerarios seguidos por las y los sujetos como secuencias, etapas o fases en la vida de un individuo. Estas etapas permiten dar cuenta de las posiciones que logra ocupar cada individuo en el campo social, las actividades desarrolladas, las instituciones en las que se desempeñan y el papel que juegan para sostener sus posiciones. De esta manera, el análisis de las trayectorias supone la consideración de aspectos estructurales, institucionales y subjetivos que dan cuenta de esas posiciones y de las condiciones y decisiones a ellas vinculadas: "lleva a elaborar la noción de trayectoria como serie de las posiciones sucesivamente ocupadas por un mismo agente (o un mismo grupo) en un espacio en sí mismo en movimiento y sometido a incesantes transformaciones" (Bourdieu, 1997).

¿Cuáles son algunas de las cuestiones involucradas en las trayectorias formativas y profesionales de los docentes y de quienes ocupan cargos directivos?

En primer lugar, los aspectos estructurales entre los cuales se incluyen la regulación del mercado laboral que, en este caso específico, son los estatutos docentes; la organización del sistema educativo en niveles, ciclos, orientaciones y asignaturas; los modos de acceso al trabajo, la carrera docente, el esquema de movilidad propuesto para el ascenso y permanencia; la estructura salarial; la organización de la formación: diseños curriculares, planes y programas; la dimensión de las políticas: los modos en que se denota y califica la docencia y a los docentes; la existencia (o ausencia) de programas de posgrado o iniciativas que 
tengan por objeto la formación continua; el contexto social, político y económico; el sistema de gobierno (centralizado, federal).

En segundo lugar, se encuentran los aspectos institucionales que, como se señaló también inciden en el ejercicio de los puestos de trabajo, es su contexto más inmediato. Como parte de esta dimensión pueden señalarse los tipos de gestión, las modalidades, los modelos organizacionales, otros pares y colegas, las dinámicas locales, prácticas que se internalizan en el habitus profesoral de cada comunidad educativa.

Finalmente, y con una incidencia no menor, se hallan también los aspectos subjetivos: el abanico de elecciones y capacidades personales, modos de ser y de relacionarse, inquietudes y deseos, limitaciones, elecciones, satisfacciones, aspectos que habilitan a permanecer en los mismos lugares o a buscar permanente otros horizontes; también los atributos personales como la edad, el sexo, o el origen socio cultural.

Todas estas dimensiones se reúnen, se combinan y se inciden mutuamente para que las trayectorias formativas y profesionales asuman determinados modos.

\section{Aspectos metodológicos del estudio y precisiones sobre el universo de análisis}

Como se señaló en la introducción, en este trabajo se presenta uno de los ejes del estudio sectorial mencionado, cuyo objetivo general fue realizar una caracterización de la oferta de educación superior tecnológica en los países participantes del proyecto, desde una perspectiva comparada, que funcione como insumo para la construcción de la agenda regional de políticas educativas para la temática.

Las ofertas de educación superior técnica (o educación terciaria técnica) que se recortan en este estudio son aquellas que cumplen los criterios establecido para los programas Educación terciaria de ciclo corto (CINE 5) según la Clasificación Internacional Normalizada de Educación 2011. Los programas de nivel CINE 5 (educación terciaria de ciclo corto) suelen estar destinados a impartir conocimientos, habilidades y competencias profesionales y se caracterizan por estar basados en un 
componente práctico, orientados a ocupaciones específicas y por preparar a los estudiantes para el mercado laboral. El nivel CINE 5 tiene una duración mínima de dos años y suele tener (aunque no siempre) una duración inferior a tres años.

Más allá del esquema organizacional que cada país adopta para la provisión de la EFTP, la educación superior o terciaria técnica está atravesando un proceso de consolidación como oferta educativa con un entorno institucional propio e independiente del modelo universitario que, en muchos casos, se funda en base a ofertas e instituciones de formación profesional que fueron promovidas al nivel terciario.

Esto evidencia que la diferenciación vertical de la oferta ha superado el binarismo en la creciente necesidad de ampliar las oportunidades y garantizar la continuidad de los estudios universitarios. Esta diversificación institucional —característica que se observa tanto entre los países como al interior de cada uno de ellos- tensiona la propia denominación del nivel. Cada vez resulta más claro que los límites que separan a estos dos ámbitos institucionales (universidades e institutos) son flexibles y cambiantes en cada sistema nacional (Jacinto, 2013).

En síntesis, la diversificación y expansión de los sistemas de educación superior puede interpretarse como respuesta ante la creciente masificación del nivel, pero también como parte del dinamismo de la EFTP. Este fenómeno se manifiesta en: a) la creación de instituciones de educación terciaria con programas de ciclo corto que, por sus objetivos y su mayor vinculación con la producción y los intereses de los territorios, resultan pertinentes y diferenciadas organizacionalmente respecto de las universidades tradicionales y b) la reducción del tiempo necesario para el egreso de los estudiantes de las universidades mediante la creación de los llamados niveles intermedios de titulación.

Según el informe de la OREALC/UNESCO (2013) en ninguno de los países estudiados se detectaron Sistemas de Información (SINFO) de la Educación y Formación Técnica y Profesional y del total de su oferta. Si bien se realizan operativos censales del sistema educativo en general, que relevan datos de la educación técnica del nivel secundario y superior técnico, no se integra con la información recogida por el sector o subsistema universitario. 
El citado informe "Estrategia para la enseñanza y formación técnica y profesional (EFTP) (2016-2021)", sostiene que dicha limitación está dada fundamentalmente por dos motivos: la presencia de múltiples actores y la ausencia de organismos responsables de fijar y reglamentar acciones de recolección de datos para el conjunto de ofertas y oferentes de programas y certificaciones de educación terciaria de ciclo corto. Esta situación se ve agravada en países como Uruguay o Paraguay en los que se vienen implementando cambios en relación con la provisión de este tipo educación (UNESCO, 2016), o como en el caso de Argentina, que cuenta con más de un organismo o dependencia a cargo de las instituciones de educación superior.

Considerando el estado de situación descripto, es posible comprender que en los cinco países estudiados el mapa institucional y de titulaciones resulte heterogéneo. Esto es así porque en cada uno de ellos existen distintas titulaciones asociadas a la EFTP, con programas que van desde los dos hasta los cinco años de duración, pudiendo estos últimos conducir a grados equiparables a los otorgados en el sector universitario. A esto se suma la dimensión institucional, ya que, a diferencia de la oferta universitaria tradicional, la educación terciaria técnica se imparte en un conjunto variado de instituciones de naturaleza jurídica distinta, asociada al tipo de titulaciones que están habilitadas a ofrecer (UNESCO, 2016).

Todos estos son elementos particulares del nivel superior técnico, aunque los datos disponibles no parecen suficientes para realizar un estudio exhaustivo de tipo regional y comparado. Según el relevamiento efectuado, los países que forman parte de este estudio no cuentan con sistemas de información que den cuenta de la organización, gestión, oferta y seguimiento de la EFTP en el nivel superior. Si bien en algunos países (como Argentina, Brasil y Chile) se detectaron avances en términos de producción y publicación de información, los restantes presentan importantes limitaciones. De este modo, la diversidad presente a nivel institucional y a nivel de los programas y certificaciones, sumada a las limitaciones de las fuentes de información oficiales, dificulta la comparabilidad entre países y estrecha los márgenes para una caracterización a nivel macro de la educación superior técnica mediante el análisis de fuentes secundarias. 
Desde esta situación de partida, se decidió utilizar una metodología de corte cualitativo, centrada en el estudio de casos. La perspectiva cualitativa sostiene la especificidad de las ciencias sociales. Aboga por el análisis de lo individual y lo concreto, por medio de la comprensión o interpretación de los significados intersubjetivos de la acción social. Es decir, desde este abordaje se intenta captar la definición de la situación que efectúan los propios actores y el significado que éstos otorgan a sus prácticas cotidianas. A su vez, se apoya en un método comparativo, cotejando casos similares entre sí pero que se diferencian también a partir de sus particularidades, buscando de formular interpretaciones que incluyan conceptos teóricos. Esta selección de casos puede incluirse dentro de lo que la metodología de la investigación denomina "estudio de casos colectivo" dado que, al mismo tiempo que permite una descripción densa de cada caso alberga la comparabilidad con fines interpretativos. La selección de los casos no se realiza al azar, sino que es producto del conocimiento de las unidades seleccionadas y de sus especificidades (Marradi et al., 2007).

La selección de los casos de estudio se llevó a cabo en el momento de diseñar la propuesta de investigación que fue oportunamente presentada en la mencionada convocatoria NEIES 2018, del estudio sectorial de referencia. Para ello, se evaluó, con los referentes de cada país, la viabilidad del desarrollo del estudio en cada institución, la anuencia de las autoridades para participar del mismo y las posibilidades concretas de realización del trabajo de campo en cada uno de los países. Considerando estos criterios, se definió la participación de instituciones de Argentina, Chile, Brasil y Uruguay.

En función de ello, los tres casos propuestos para el estudio fueron:

- el Instituto Tecnológico Beltrán (Argentina),

- el Instituto Profesional Los Lagos (Universidad de los Lagos, Chile),

- y la experiencia de los Cursos Binacionales impartidos por el Consejo de Educación Técnico Profesional - Universidad del Trabajo de Uruguay (CEPTUTU, Uruguay) y el Instituto Federal de Educación, Ciencia y Tecnología Sulrio-grandense (IFSul, Brasil). 
Como se mencionó, dentro del enfoque propuesto para este estudio se utilizaron diferentes métodos de recolección a efectos de aumentar la validez de los resultados y el grado de confianza en los mismos (Campbell y Fiske, 1959). En las ciencias sociales esta estrategia de investigación se denomina "triangulación", dado que combina diferentes métodos para favorecer la adquisición de un conocimiento más amplio y profundo de la realidad social (Denzin, 1989).

El trabajo de campo tuvo lugar en 2019. Se realizaron entrevistas semiestructuradas y en profundidad que permitieron conocer la realidad desde la perspectiva de los propios responsables de política de EFTP, a efectos de describir e interpretar aspectos que no son directamente observables en los documentos y la normativa: impresiones, opiniones, intenciones, proyectos, etc. El carácter semiestructurado de las entrevistas implica, como ha sido mencionado, que se adecuaron las preguntas iniciales a las respuestas que el entrevistado iba proporcionando.

En total se realizaron 52 entrevistas (entre individuales y grupales) con informantes clave de las experiencias consideradas para el estudio y se obtuvieron 29 registros de trayectorias formativas y profesionales, captados a través de un instrumento auto-administrado y específicamente diseñado para tal fin. El trabajo de campo abarcó a un conjunto amplio de actores de diferentes ámbitos vinculados a las experiencias visitadas:

- Referentes del gobierno municipal/departamental

- Director/Rector de la institución

- Asesores y otros miembros del equipo directivo de la institución

- Coordinadores/Directores Académicos de la institución

- Jefes de Departamento/Jefes de Carrera de la institución

- Docentes de la institución

- Alumnos de la institución

- Referentes del ámbito empresarial

Para resguardar las normas éticas de la producción académica se preserva la identidad de las personas entrevistadas, quienes generosamente destinaron tiempo de trabajo y personal para los intercambios que tuvieron lugar en el marco del 
proyecto. Asimismo, la utilización de citas textuales se realizó sin identificación de individuos, mencionando solamente a su perfil, cargo o función para contextualizar sus testimonios. Todas las entrevistas se realizaron en los ámbitos de inserción laboral, toda vez que el estudio abordó también el componente de análisis institucional. Las instituciones sí son identificadas porque constituyen ejemplos arquetípicos, criterio que formó parte del proceso de selección y que, por eso, se convirtieron en "casos" de interés para la investigación.

Para complementar la indagación se efectuaron consultas con especialistas en EFPT en los países seleccionados, perfiles académicos y funcionarios con reconocida trayectoria en el estudio y la gestión de estos sistemas educativos. Sus aportes resultaron relevantes para reconstruir los debates sobre la situación actual de los sistemas de EFTP en Latinoamérica, así como también para delinear las tendencias a futuro en los países bajo estudio. Finalmente, entre los meses de julio y noviembre de 2019 se realizaron entrevistas con expertos internacionales del ámbito académico, las cuales tuvieron un carácter abierto y su propósito fue ampliar la mirada respecto de los hallazgos parciales encontrados en el estudio.

\section{Las trayectorias formativas y profesionales de los integrantes de los equipos directivos}

Este apartado presenta el análisis de los principales aspectos comunes y divergentes relativos a las dimensiones bajo análisis: trayectoria profesional, formativa y desempeño en el cargo. Hacia el final se presentan algunas reflexiones más generales sobre las condiciones de los equipos directivos en las instituciones que fueron objeto de análisis.

A partir de los casos de estudio de Argentina (Instituto Beltrán), Chile (Instituto Profesional Los Lagos) y Brasil/Uruguay (Cursos Binacionales) se propone una lectura en clave comparativa de los principales temas emergentes en cada una de las dimensiones consideradas, buscando poner de relieve tanto las similitudes encontradas como también las particularidades y rasgos distintivos de cada experiencia, desde la perspectiva conceptual propuesta en los puntos de partida. 


\subsection{Trayectoria profesional y formativa}

Al analizar los recorridos profesionales de los 32 integrantes de los equipos directivos alcanzados por esta indagación, se puso de relieve que la gran mayoría ha tenido un amplio recorrido en la docencia, incluso en instituciones educativas de otros niveles (mayormente de nivel secundario y universitario). La experiencia adquirida como docentes fue ampliamente valorada para el desarrollo de las tareas vinculadas a la gestión educativa. En todas las instituciones, la necesidad de acompañamiento al cuerpo docente, el apoyo en cuestiones pedagógicas y didácticas, así como también el impulso para el desarrollo de nuevos proyectos áulicos, se destacaron como parte de las actividades llevadas a cabo por los equipos directivos.

Por otro lado, en cuanto a la experiencia profesional en el medio socioproductivo, a excepción de los gestores de la UTU en Uruguay, los integrantes de Ios equipos de Argentina, Brasil y Chile afirmaron poseer una amplia trayectoria, ya sea en el ejercicio de su profesión de base o directamente en industrias y empresas de servicios. En los tres casos, se hizo mención explícita a los perfiles requeridos por los institutos: docentes y directivos que tengan experiencia de trabajo en el mundo productivo. Puede leerse en esta condición la importancia que tiene para esta oferta formativa el vínculo con el ámbito laboral.

En el caso de Brasil, la modalidad contractual de dedicación exclusiva con el instituto ha implicado, en muchos casos, la interrupción del trabajo en medio socioproductivo, mientras que, en los casos de Argentina y Chile, fue posible la coexistencia de ambas funciones. El impacto que genera la trayectoria profesional del cuerpo docente y del equipo directivo en las instituciones formadoras fue resaltado positivamente por todos los integrantes del Instituto Beltrán, del Instituto Profesional Los Lagos y del IFSul. Cabe señalar en este sentido dos aspectos que emergen con consistencia en los relatos. En primer lugar, los consultados afirmaron que su vinculación con el medio socio-productivo los mantuvo permanentemente actualizados en relación con las tendencias tecnológicas y las modalidades de trabajo implementadas en el sector. Esa cercanía con el mundo laboral les permitió enriquecer la labor docente incorporando ejemplos significativos en las clases o bien 
utilizando análisis de casos reales ${ }^{3}$. Asimismo, les permitió establecer una relación más clara entre los conceptos teóricos y las habilidades prácticas que buscan desarrollar en los estudiantes, pudiendo así orientar mejor la labor de otros docentes. En segundo lugar, la experiencia de trabajo en el mundo socio-productivo mejoró su desempeño como gestores de instituciones educativas. Según las menciones registradas, los consultados afirmaron que esta experiencia colaboró con una planificación más eficiente de las tareas y procedimientos, mejoró sus resultados en los casos de trabajo bajo presión y facilitó el trabajo en equipo.

En cuanto a la experiencia de trabajo en ámbitos gubernamentales, en todos los países se registraron menos referencias al respecto, es decir que la mayor parte de los gestores y directivos consultados afirmaron no tener experiencia de trabajo en este ámbito. Sin embargo, esa porción minoritaria de casos que sí se ha desempeñado en dependencias de gobiernos locales y/o ministerios nacionales o provinciales, destacó que el impacto de la experiencia en su desempeño actual fue positivo. Los efectos se evidenciaron principalmente en una ampliación de las redes de contactos que facilitó desde la incorporación de nuevos perfiles docentes a los institutos, hasta la simplificación de gestiones administrativas. A su vez, la experiencia en ámbitos de gestión central otorgó a los consultados una visión más amplia de la planificación de las políticas públicas y de las diversas situaciones a las que las mismas deben atender. En este sentido, su paso por ámbitos centrales de gobernanza les aportó herramientas para comprender los posibles desfasajes entre aspectos normativos o regulatorios y las particularidades propias de las instituciones que dirigen.

Otro de los emergentes surgidos del relevamiento está vinculado con la identificación de aquellas situaciones que fueron motivadoras para el desarrollo de las tareas de gestión y dirección de instituciones superiores. En este punto, surgieron dos grandes coincidencias que atravesaron los cuatro casos de estudio.

\footnotetext{
${ }^{3}$ En este punto, es importante recordar que parte de los miembros de los equipos directivos continúan realizando actividades docentes, el aula no es algo que han abandonado para desarrollar la función de conducción. Es decir que también se valora el vínculo con los y las estudiantes en formación.
} 
En primer lugar, gran parte de los actores consultados hicieron mención a gestos de confianza por parte de sus superiores que implicaron la delegación de funciones y de nuevas responsabilidades. Así, el aval para coordinar y desarrollar proyectos innovadores o asumir tareas y responsabilidades desafiantes, fueron destacadas como fuente de motivación para el desarrollo del cargo. Este rasgo también da cuenta del circuito de reclutamiento que suele establecerse en las instituciones para dar continuidad a proyectos institucionales, promoviendo la inclusión del personal cercano a los equipos directivos y con posibilidades de sostener las líneas de trabajo en virtud de las capacidades que son identificadas por quienes están a cargo de las instituciones. Asimismo, las menciones relativas al progreso y las mejoras de la situación profesional y personal de los alumnos de los institutos fueron consideradas como un importante motor de la actividad realizada.

A pesar de que varios de los integrantes de los equipos de conducción señalaron tener experiencia docente previa o simultánea, se mencionó como necesaria la importancia del acompañamiento, guía y actualización en cuestiones pedagógicas y didácticas - especialmente en los casos de Brasil y Argentina-. A su vez, se detectó una baja presencia de cursos específicos para la formación directiva o la actualización en aspectos particulares de la educación superior, validando las primeras afirmaciones que inician este capítulo. Por otro lado, a excepción de los integrantes de los equipos de Argentina, gran parte de los directivos destacaron enfáticamente la necesidad de realizar capacitaciones enfocadas al desarrollo de habilidades blandas como: liderazgo y resolución de conflictos, técnicas de negociación y manejo de crisis, comunicación y relaciones interpersonales.

\subsection{Desempeño en el cargo}

Una de las cuestiones abordadas en esta dimensión se enfocó en la identificación de aspectos desafiantes en la gestión de instituciones superiores. En este punto, dos aspectos surgieron con fuerza del relato de los entrevistados. En primer lugar, en los casos de Chile, Brasil y Uruguay, uno de los desafíos mencionados fue sostener la motivación del cuerpo docente para realizar sus tareas. Si bien la valoración del equipo docente fue en todos los casos muy positiva, 
destacando su alto grado de formación y su nivel de compromiso, desde las funciones directivas se evidenció un trabajo sostenido para que los docentes se impliquen en las actividades de los institutos. A su vez, especialmente en los casos de Brasil y Chile, se mencionó como un desafío para los equipos directivos el buen manejo de las relaciones interpersonales, la resolución de situaciones de conflicto e intereses contrapuestos y el ejercicio de liderazgo por parte de los directores.

Por otro lado, los institutos de Chile, Brasil y Uruguay coincidieron en mencionar como un desafío el sostenimiento de las relaciones con las unidades centrales de gestión. Comentarios acerca de un "diálogo difícil entre lo local y lo nacional", al "olvido" por parte de las dependencias nacionales de las cuales se encuentran alejados territorialmente y las dificultades para gestionar una institución dispersa territorialmente - como es el caso de IFSuL - pusieron de manifiesto las tensiones surgidas de la descentralización de las políticas educativas. Como contracara, en todos los casos se encontraron referencias a las buenas relaciones que han podido establecer los institutos con sus comunidades locales, para lo cual el desempeño del equipo directivo fue de suma relevancia. De este modo, puede observarse el interés que estas instituciones tienen en el vínculo con las comunidades más cercanas y los esquemas de funcionamiento que sostienen con las estructuras centralizadas. En este punto es de destacar también la desigual presencia de aquellas instituciones que tienen un andamiaje institucional más robusto o que integran una red de instituciones (IFSul de Brasil) o de aquellas que tienen además vínculos con otros organismos para sostenerse (Instituto Beltrán).

En cuanto a la identificación de las dificultades aparejadas a la gestión de instituciones de educación superior, tanto en Chile como en Brasil y Uruguay, se mencionó la insuficiencia o la merma de recursos financieros. Si bien los entrevistados señalan que en gran medida cuentan con los recursos para realizar sus actividades, al momento de identificar las dificultades para la gestión de las instituciones surgieron menciones concretas a la escasez de recursos financieros y a la falta de cargos o puestos requeridos. Asimismo, se destacaron las referencias a las gestiones que, tanto los directivos como los docentes, han realizado para sostener y mejorar las actividades realizadas, ampliar las fuentes de financiamiento 
y generar convenios de cooperación e intercambio con empresas y otras instituciones. En este punto, la trayectoria de los directivos, sus redes de contacto y sus vinculaciones con otras organizaciones y con el medio socio-productivo, implicaron un diferencial para el funcionamiento de los institutos.

Otro tópico relevante de mencionar son los atributos percibidos como necesarios para desempeñarse en la función directiva de una institución de educación superior. De un total de 79 respuestas señaladas, un $76 \%$ hizo referencia a atributos personales vinculados con las relaciones interpersonales y el carácter de los directivos; mientras que el $24 \%$ mencionó atributos correspondientes a la formación académica y la trayectoria profesional en el cargo. Entre las características y atributos personales, los aspectos más mencionados fueron los siguientes: Buenas habilidades interpersonales, empatía, capacidad de escucha (17 menciones); Liderazgo y carisma (17 menciones); Capacidad de resolución, poder de decisión (7 menciones); Capacidad de trabajo en equipo (6 menciones); Responsabilidad y compromiso con la función (6 menciones); Buena comunicación, motivación (4 menciones); Flexibilidad (2 menciones).

Entre los tópicos ineludibles para el trabajo directivo se señala la comunicación organizacional y destinada a diferentes actores (familias, estudiantes, autoridades municipales, prensa), la planificación y la gestión, el carácter, la necesidad de tener experiencia en formación docente, y la experiencia o el conocimiento dado por la práctica.

En el marco de este estudio interesa también identificar las áreas en las que es requerida algún tipo de formación específica para el desempeño profesional de quienes ocupan cargos directivos. Los temas mayormente mencionados fueron: conocimientos de gestión y administración de instituciones educativas (8 menciones); experiencia en dirección de instituciones de educación superior (4 menciones); herramientas de planificación ( 3 menciones) y formación docente ( 2 menciones). Sobre este punto, también es necesario señalar el aparente desplazamiento desde un modelo de dirección institucional fundamentado en las habilidades de la planificación tradicional o de la planificación estratégica como herramientas fundamentales para gestionar y coordinar una institución educativa, 
hacia un modelo centrado en el requerimiento de un conjunto de habilidades "blandas" y de valores, en el que la dimensión vincular surge como el eje del trabajo que han de enfrentar los nuevos perfiles directivos.

La función directiva, leída en clave de trayectoria y de desempeño profesional puede comprenderse también como una actividad que se construye en el camino, en colaboración con otros colegas y que aun siendo una tarea de mucha responsabilidad resulta ser un ámbito valorado y disfrutado por quienes están a cargo de las tareas de dirección y coordinación de las instituciones analizadas. Del mismo modo, el modelo organizacional y la propuesta formativa requieren ser estudiados considerando también los perfiles de quienes tienen a su cargo ambas tareas, la de conducción y la de docencia o de acompañamiento de las funciones docentes.

\section{A modo de cierre}

Del análisis realizado a partir de los registros de 29 cuestionarios aplicados a integrantes de equipos directivos de las instituciones de los países bajo análisis, hay algunos rasgos destacables en relación con las trayectorias formativas. En todos los países, quienes ocupan esos puestos han accedido a estudios superiores, situación que refleja también la ampliación del acceso a estudios en los países de la región y la profesionalización de las áreas de gestión. El acceso a estudios de posgrado, si bien está presente, no está tan extendido. Muchos de los entrevistados han realizado cursos o formaciones de posgrado en temas relativos a la gestión institucional, aunque no de modo específico para desempeñarse en instituciones de educación superior. En algún caso se hace referencia (Chile) a una formación realizada en París, de la que participaron varios integrantes de la institución, instancia valorada en tanto trabajo colectivo que luego ha sido capitalizado para acciones institucionales específicas.

En relación con las trayectorias profesionales, muchas de las personas entrevistadas señalan haber tenido inserciones previas en el sector privado y en ámbitos vinculados a diferentes sectores productivos. Otro conjunto ha tenido participación en ámbitos gubernamentales de nivel nacional/federal, municipal y 
finalmente, otros han tenido desempeños previos en el sistema educativo, en universidades o en otros niveles de enseñanza. Todas las instituciones dan cuenta de un trabajo que se realiza en equipo, que incluye reuniones de trabajo, agendas compartidas, modos colaborativos para el desarrollo de proyectos e intervenciones específicas y, en algunos casos, esas tareas incluyen también espacios de formación en temáticas específicas o jornadas de actualización profesional. Dependiendo del armado institucional de cada uno de los modelos institucionales, hay una mayor presencia de trabajo por proyectos o con seguimientos más formalizados o rutinizados que moldea el tipo de tareas que estos perfiles desarrollan.

Otro de los aspectos abordados en este eje de análisis tuvo por objetivo relevar algunos de los temas que se identificaron como carencias de la formación y aspectos que serían necesarios para el desempeño en los cargos de conducción. Hay dos tipos de temáticas señaladas en general por las personas entrevistadas: aquellas cuestiones que pueden identificarse como más vinculadas a "habilidades blandas" y aquellas más características de ámbitos de planeamiento y gestión al modo tradicional. Algunos de los contenidos señalados quedan recogidos en el Gráfico 1.

\section{Gráfico 1. Principales temas de capacitación identificados por los entrevistados}

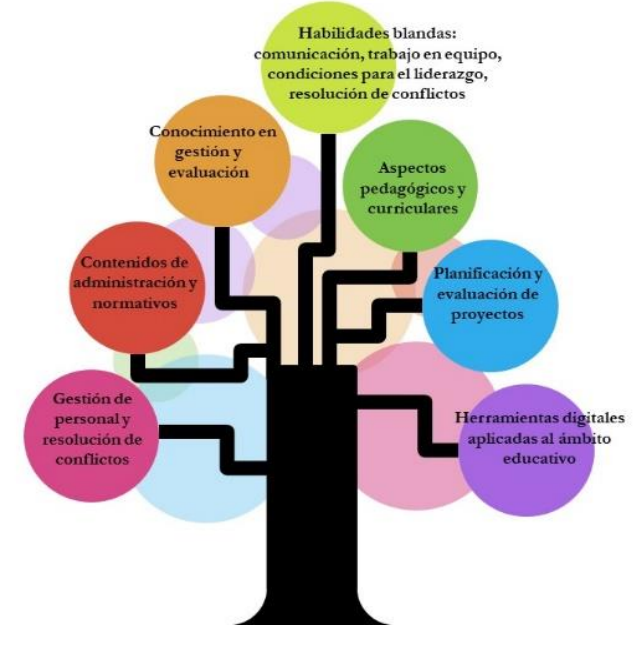


Por último, uno de los tópicos surgidos del relevamiento está vinculado con la identificación de aquellas situaciones que fueron motivadoras para el desarrollo de las tareas de gestión y dirección de instituciones superiores. En este punto, surgieron dos grandes coincidencias que atravesaron los cuatro casos de estudio. En primer lugar, gran parte de los actores contactados hicieron mención a gestos de confianza por parte de sus superiores que implicaron la delegación de funciones y de nuevas responsabilidades. Así, el aval para coordinar y desarrollar proyectos innovadores o asumir tareas y responsabilidades desafiantes, fueron mencionadas como fuente de motivación para el desarrollo del cargo. Asimismo, las menciones relativas al progreso y las mejoras de la situación profesional y personal de los alumnos y alumnas de los institutos fueron consideradas como un importante motor de la actividad realizada. Este rasgo también da cuenta del circuito de reclutamiento que suele establecerse en las instituciones para dar continuidad a proyectos institucionales, promoviendo la inclusión del personal cercano a los equipos directivos y con posibilidades de sostener las líneas de trabajo en virtud de las capacidades que son identificadas por quienes están a cargo de las instituciones.

El conjunto de aspectos señalados como necesarios para la formación aporta a la posibilidad de organizar una agenda formativa que tenga como destinatarios a quienes se desempeñan en estos espacios de trabajo en una oferta que, como se señaló al inicio del trabajo, flexibiliza sus propuestas para responder a los desafíos sociales y formativos del mundo contemporáneo.

\section{Referencias bibliográficas}

Alliaud, A. (2004). La biografía escolar en el desempeño profesional de los docentes noveles. Tesis de Doctorado. Universidad de Buenos Aires, Facultad de Filosofía y Letras.

Alliaud, A. (2010). La formación en y para la práctica profesional. Conferencia realizada el 19 de agosto en Ciudad de Buenos Aires, INFD, Ministerio de Educación. 
Bourdieu, P. (1997). Razones prácticas, sobre la teoría de la acción. Barcelona: Anagrama.

Briasco, I., Botinelli, L. y Montes, N. (2020) La oferta de educación terciaria tecnológica. Caracterización de los modelos de gestión institucional desde la perspectiva de los estudios comparados (Informe Final de Estudios Sectoriales). Inédito. Buenos Aires: NEIES. SEM.

Campbell, D. y Fiske, D. (1959). Convergent and discriminant validation by the multitrait multimethod matrix. Psychological Bulletin, 56 (2), 81-105.

Denzin, N. (1989). Strategies of Multiple Triangulation. The Research Act: $A$ theoretical Introduction to Sociological Methods. New York: Mc Graw Hill.

Jacinto, C. (2013). La educación post-secundaria técnica: contexto, interrogantes y aportes de la investigación. En Jacinto (Comp.), Incluir a los jóvenes. Retos para la educación terciaria técnica en América Latina (pp. 39-66). Paris: IIPE-UNESCO. Disponible en https://bit.ly/3ofUWbk

Marradi, A., Archenti, N. y Piovani, J.I. (2007). Metodología de las Ciencias Sociales. Buenos Aires: Emecé Editores.

Montes, N. y Sendón, M. (2006). Trayectorias educativas de estudiantes del nivel medio en la Argentina a comienzos del siglo XXI. Revista Mexicana de Investigación, 11 (29), 381-402.

OEI (2017). Miradas sobre la educación en Iberoamérica. Desarrollo profesional y liderazgo de directores en Iberoamérica. Madrid: IESME.

OREALC/UNESCO (2013). Estado de situación de los Sistemas de Información (SINFO) de la Educación y Formación Técnica y Profesional (EFTP) en 12 países de América Latina y el Caribe. Santiago de Chile: OREALC/UNESCO. Disponible en https://bit.ly/34dMe5g

Rivas Flores, J. (2007). Vida, experiencia y educación: la biografía como estrategia de conocimiento. En I. Sverdlick (Coord.), La investigación educativa. Una herramienta de conocimiento y de acción (pp. 111 a 146). Buenos Aires: Noveduc.

Terigi, F. (2010). Desarrollo profesional continuo y carrea docente en América Latina. Santiago de Chile: PREAL. 
UNESCO (2015). Recomendación Relativa a la Enseñanza y Formación Técnica y Profesional (EFTP). Paris: UNESCO. Disponible en https://bit.ly/37mCOGC UNESCO (2016). Estrategia para la enseñanza y formación técnica y profesional (TVET) (2016-2021. Paris: UNESCO. Disponible en https://bit.ly/31JI9UX UNESCO (2018). La Enseñanza y Formación Técnico Profesional en América Latina y el Caribe. Una perspectiva regional hacia 2030. Santiago de Chile: UNESCO. Disponible en https://bit.ly/31sb2EF

Veleda, C. (2016). ¿Una bala de plata para mejorar la calidad educativa? La formación de los supervisores y directores de escuela. Buenos Aires: CIPPEC.

Weinstein, J., Muñoz, G. y Hernández, M. (2014). El liderazgo directivo escolar en América Latina y el Caribe. Un estado del arte con base en ocho sistemas escolares de la región. OREAL-UNESCO.

Fecha de recepción: $\mathbf{2 6}$ de agosto de $\mathbf{2 0 2 0}$ Fecha de aceptación: 23 de octubre de 2020

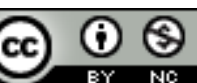

Revista Educación, Política y Sociedad (ISSN 2445-4109) está distribuida bajo una Licencia Creative Commons Atribución-NoComercial 4.0 Internacional 